REPORT

\title{
Functions of AP1 (Fos/Jun) in bone development
}

\section{E F Wagner}

Ann Rheum Dis 2002;61 (Suppl II):ii40-ii42

Genetically modified mice and cells have provided important insights into the biological functions of the dimeric transcription factor complex AP1, in particular into its role in skeletal development. Data obtained from knockout mice revealed that some components, such as c-Fos are key regulators of bone cell differentiation, whereas others, like c-Jun, JunB and Fra-1 are essential in embryonic and/or postnatal development. Apart from identifying the specific roles of API proteins in developmental processes, researchers are beginning to obtain a better molecular understanding of their cell-context dependent functions, their downstream target genes and how they regulate bone cell proliferation, differentiation, and apoptosis.

A great variety of dimers composed of members of the Fos, Jun, and ATF families of proteins constitute the transcription factor complex APl (for review see references ${ }^{12}$ ). While the Fos proteins (c-Fos, FosB, Fra-1, Fra-2) can only heterodimerise with members of the Jun family, the Jun proteins (c-Jun, JunB, JunD) can both homodimerise and heterodimerise with Fos members to form transcriptionally active complexes. Some members of the ATF and CREB families of proteins are also part of API complexes. APl converts extracellular signals into changes in the expression of specific target genes, which harbour APl binding site(s) in their promoter or enhancer regions. The activity of APl is modulated by interactions with other transcriptional regulators and is further controlled by upstream kinases that link APl to various signal transduction pathways. APl has been implicated in a large variety of biological processes including cell differentiation, proliferation, apoptosis, and oncogenic transformation. Here I will summarise the current understanding of APl functions in bone development, which was largely obtained from the analysis of genetically modified mice and cells in which specific APl genes have been ectopically expressed, inactivated, mutated, or replaced by each other.

Most important insights regarding the specific functions of APl proteins in mouse development were obtained from loss of function experiments using ES cell technology. A summary of the phenotypes of mice harbouring genetic modifications of the different fos and jun genes is given in table 1. These analyses showed that APl proteins have specific functions during embryogenesis and organogenesis. With regard to embryonic development, the Fos and Jun proteins can be grouped into two categories: some such as c-Fos, FosB, and JunD are dispensable, whereas others like c-Jun, JunB and Fra-l are essential for embryonic development. In the following I will discuss the specific phenotypes of knock out mice in particular of c-Fos and Fra-l as well as studies in transgenic mice expressing individual APl components with an emphasis on the effects observed on bone development.

\section{API IN BONE DEVELOPMENT}

The growth and the maintenance of the skeleton depends on the coordinated function of osteoblasts and osteoclasts, the two principal cell types of bone tissue. ${ }^{34}$ Osteoblasts, which derive from mesenchymal progenitors, produce the extracellular matrix of the bone that later undergoes mineralisation. In contrast, osteoclasts belong to the monocyte/macrophage lineage and reduce bone mass by resorbing the mineralised extracellular matrix. Recent studies have shown that APl components, mainly members of the Fos family such as c-Fos and Fra-1, have important functions in both of these cell types.

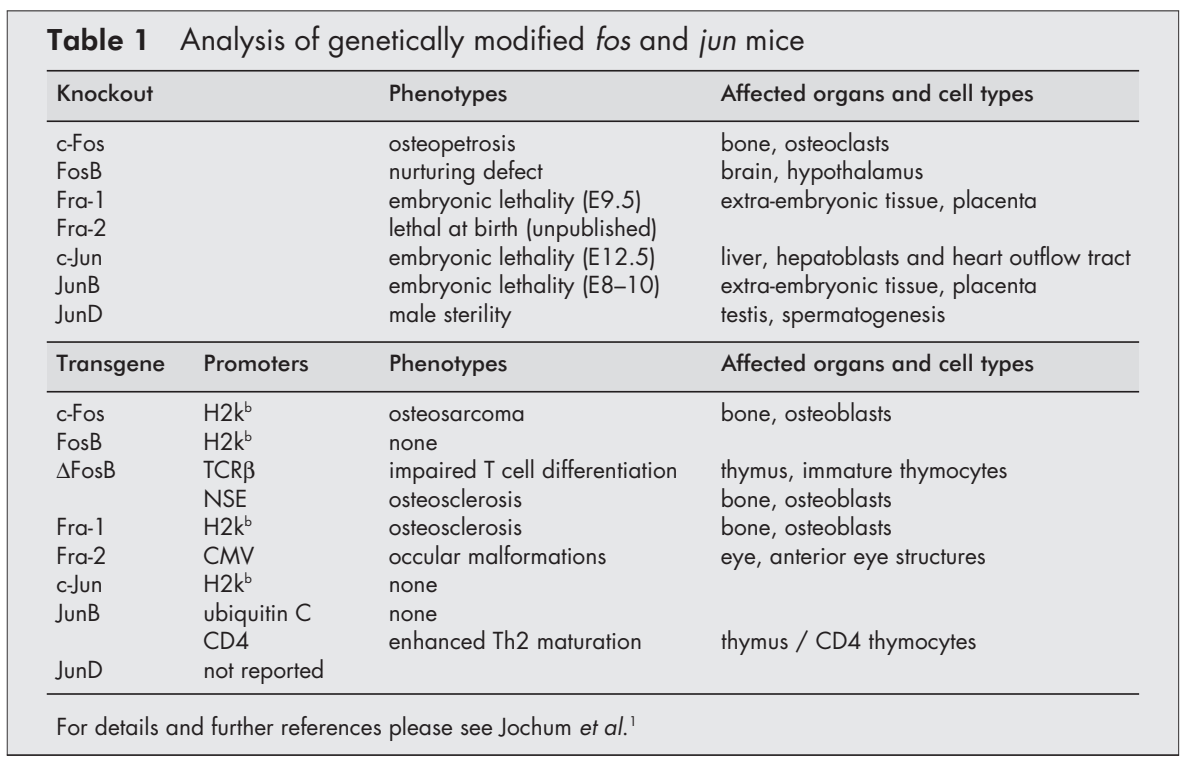




\section{Osteoblasts and the role of c-Fos and Fra-1}

In osteoblasts, APl activity can be induced by transforming growth factor $\beta$, parathyroid hormone and 1,25-dihydroxy vitamin $\mathrm{D}$, which are potent regulators of osteoblast differentiation and proliferation. ${ }^{4}$ The various members of the APl complex are differentially expressed during osteoblast maturation in vitro ${ }^{5}$ with all Fos and Jun proteins initially being highly expressed. Subsequently, during the period of extracellular matrix production and mineralisation, their levels decline, and Fra-2 and JunD become the major components of the APl complex in fully differentiated osteoblasts. The pattern of c-Fos expression during development suggests a critical role in endochondral ossification, ${ }^{6}$ although the analysis of c-Fos deficient mice indicates that c-Fos is dispensable for the differentiation of osteoblasts. ${ }^{78}$

When c-Fos was ectopically expressed in various cell types and in transgenic mice, specific effects on the skeleton were observed. Chimeric mice obtained from c-Fos overexpressing embryonic stem cells develop chondrogenic ${ }^{9}$ tumours implying a function of c-Fos in chondrogenesis in vivo. Surprisingly, c-Fos overexpression in an in vitro model of chondrogenesis inhibits the differentiation of chondrocytes. ${ }^{10}$ Ectopic c-Fos expression from a ubiquitous promoter in transgenic mice has no noticeable effects on cell differentiation in most organs, but results in the specific transformation of osteoblasts leading to osteogenic sarcomas reminiscent of human osteosarcomas. ${ }^{11}$

Importantly, transgenic mice overexpressing Fra-1 in osteoblasts as well as in other cell types exhibit again a specific bone phenotype. These mice show increased bone formation and develop osteosclerosis of the entire skeleton. ${ }^{12}$ This phenotype is attributable to a cell autonomous increase in the number of mature osteoblasts indicating that Fra- 1 increases osteoblast differentiation. A similar osteosclerosis phenotype is observed in transgenic mice expressing $\Delta$ FosB in osteoblasts. ${ }^{13}$ These mice also show reduced adipogenesis, a phenotype that is not observed in Fra-1 transgenic mice.

Given the osteoblastic phenotype of the Fra-l transgenic mice it was of interest to next ask whether the absence of Fra- 1 affects osteoblast or osteoclast differentiation. Inactivation of Fra-1, however, results in embryonic lethality around day 10 of development because of defects in the placenta thereby precluding the analysis on bone cell development. ${ }^{14}$ The labyrinth layer of mutant placentas is reduced in size and largely avascular suggesting that the invasion of allantoic vessels into the chorionic plate is impaired in the absence of Fra-1. The development of mutant fetuses can be rescued up to birth by providing wild type extra-embryonic tissues using tetraploid blastocyst injection. ${ }^{15}$ This suggests that Fra-l is dispensable for the differentiation along most, if not all, cell lineages in the fetus. ${ }^{14}$ Moreover, the lethality of Fra-1 deficient mice was fully rescued by the ectopic expression of Fra-1 from transgenic mice ${ }^{14}$ demonstrating that APl/Fra-1 activity during development does not have to be tightly regulated. These rescued mice still developed osteosclerosis, which was indistinguishable from the disease observed in the Fra-1 transgenic mice. However, it cannot be excluded that Fra- 1 has a critical role in osteoblast homeostasis during postnatal life. We recently demonstrated using a conditional allele of fra- 1 that mice without Fra-1 can indeed be born and develop osteopenia, which has to be further characterised (Robert Eferl, unpublished data).

\section{Osteoclasts and the role of c-Fos and Fra- 1}

Mice lacking c-Fos are viable and fertile but lack osteoclasts and are therefore osteopetrotic. ${ }^{78}$ This phenotype is strain dependent, as on a 129/sv background much fewer pups are born and reach weaning age, when compared with C57Bl/6 mutant mice (unpublished observations). Mutant mice also show abnormalities of the haematopoietic system including extramedullary haematopoiesis and lymphopenia, which are both secondary to the bone phenotype. ${ }^{16}$ Although c-Fos is rapidly induced in T cells upon activation and it regulates the transcription of various cytokines including interleukin 2, the analysis of c-Fos deficient mice has shown that c-Fos is not required for the differentiation and activity of peripheral T cells. ${ }^{17}$

The lack of osteoclasts in c-Fos deficient mice is accompanied by increased numbers of bone marrow macrophages, which is specific for this osteopetrotic mutant. The osteopetrosis is characterised by increased bone mass attributable to reduced bone resorption. ${ }^{7818}$ Recent data using retroviral gene transfer into c-Fos mutant osteoclast precursors in vitro have shown that all Fos proteins, most efficiently Fra-l, can complement for the absence of c-Fos. ${ }^{19}$ Further structurefunction analysis also demonstrated that the major C-terminal transactivation domains of c-Fos and FosB are dispensable for the rescue of osteoclast formation in vitro. Interestingly, Fra-1, which lacks a transactivation domain, has the highest rescue activity. Moreover, the osteoclast differentiation factor RANKL also known as ODF, OPGL, TRANCE (for review see Karsenty and Wagner ${ }^{4}$ ) induces besides c-Fos also Fra-l transcription in a c-Fos dependent manner, thereby establishing a link between RANK signalling and the expression of APl proteins during osteoclast differentiation ${ }^{19}$ (see also below).

The osteopetrotic phenotype can be partly cured in c-Fos mutant mice by expressing a Fra-1 transgene, one target gene of c-Fos in the osteoclast lineage. ${ }^{19}$ Importantly, total restoration of osteoclast differentiation was achieved in knock in mice generated by the insertion of the fra- 1 gene into the $\mathrm{c}$-fos locus. ${ }^{20}$ Fra- 1 was able to rescue c-Fos dependent functions, in particular bone development and light induced photoreceptor apoptosis. Interestingly, the rescue of bone cell differentiation was gene-dose dependent and Fra- 1 was unable to substitute for the expression of APl target genes in fibroblasts. These results show that c-Fos and Fra- 1 have maintained functional equivalence during vertebrate evolution and further suggest that functional diversity of Fos genes is a result of divergence of regulatory rather than protein coding sequences.

In addition to the effects on osteoblasts, ectopic Fra-1 expression also increases the differentiation of osteoclasts, both in progenitor cell lines and primary osteoclast progenitors. ${ }^{1921}$ This strong osteoclastogenic effect is not apparent in Fra-l transgenic mice in vivo. ${ }^{12}$ Osteoclast differentiation on the other hand does not seem to require the presence of Fra-1, as the skeleton of rescued Fra-1 deficient fetuses contains functional osteoclasts at birth. ${ }^{14}$ Moreover, recently established Fra- 1 knockout mice using a conditional gene targeting approach demonstrate that a normal skeleton can be formed (Robert Eferl, unpublished data and see above) suggesting that Fra-l is dispensable for the formation and differentiation of the major bone cell types, the osteoblasts and the osteoclasts.

\section{The role of c-Fos in signalling during osteoclast differentiation}

To maintain bone homeostasis the signalling by the osteoclastogenic ligand RANKL, a member of the TNF family and an essential factor for osteoclast differentiation, has to be tightly controlled, as deregulated osteoclast activity leads to severe bone diseases such as osteoporosis. ${ }^{4}$ In a genomic search for c-Fos target genes induced by RANKL in osteoclast precursor cells using wild type and c-fos-/- cells, a group of mRNAs whose expression is dependent on interferon $\alpha / \beta$ signalling was found to be specifically induced by c-Fos. The induction was decreased in cells lacking c-Fos and the down regulated genes included interferon responsive genes such as Ifit-3, Ifit-2, Mx-1, Scyb-10. and Irf-7 (unpublished and Takayanagi et $\left.a l^{22}\right)$. Although the integral role of the interferon $\alpha / \beta$ system in the regulation of immune responses has been extensively documented, it was unknown whether this system is linked to RANKL signalling and bone metabolism. We found that 


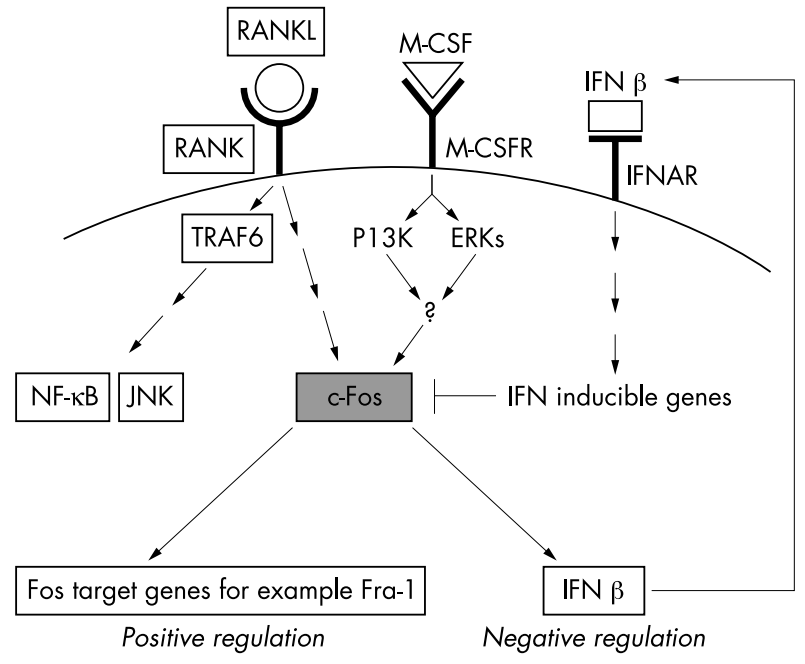

Figure 1 Signalling in osteoclast progenitor cells and cross talk with the interferon pathway. For details see also Karsenty and Wagner ${ }^{4}$.

RANKL directly induced the interferon $\beta$ gene in a c-Fos dependent manner in osteoclast precursor cells. However, interferon $\beta$ inhibits the differentiation of osteoclasts by interfering with the RANKL induced expression of c-Fos, which is essential for osteoclast formation. ${ }^{22}$ The mechanism of interferon $\beta$ gene induction by c-Fos is distinct from that induced by viruses and implies that an autoregulatory mechanism is operating in fine tuning the activity of osteoclasts. RANKL induces in the same progenitor cell c-Fos, which in turn induces the expression of positive regulators such as Fra- 1 as well as its own inhibitor interferon $\beta$ (fig 1 ). The inhibition of c-Fos by interferon $\alpha / \beta$ signalling occurs at the posttranscriptional level and the molecular mechanism has yet to be defined. The importance of this regulatory mechanism for bone homeostasis is supported by the observation that mice deficient in interferon $\beta$ signalling exhibit severe bone loss accompanied by increased osteoclastogenesis. ${ }^{22}$ Moreover, we were able to show in a model of LPS induced inflammatory bone destruction that the application of interferon $\beta$ had a therapeutic effect in this disease model. Therefore, this study may offer new approaches to the treatment of bone diseases caused by excessive osteoclastogenesis, such as autoimmune arthritis and osteoporosis.

\section{CONCLUSIONS}

Through the extensive analysis of the individual APl components using genetically modified mice and cells we are beginning to unravel the specific functions of these proteins in development and/or in the adult organism. The initial studies have largely focused on c-Fos and c-Jun, but have been extended to other family members. As the absence of c-Jun, JunB and Fra- 1 results in embryonic lethality, the analysis of these proteins during later stages of development and in postnatal life were not possible without the development of new genetic tools. With the conditional inactivation of APl genes in a cell type specific and/or inducible manner using the established Cre/loxP and tet regulated systems, novel functions are being assigned to the individual members of APl (for example, Behrens et $a l^{23}$ ). However, we still do not understand the enormous specificity of proteins such as c-Fos and Fra-l for osteoblast and/or osteoclast function. Nor do we know the individual partners of these molecules responsible for their transcriptional activity and specificity. In addition, we only have a very vague picture about the important downstream genes controlled by these factors. The genomic analysis exemplified by the discovery of the interferon $\beta$ system as a c-Fos regulated gene cascade illustrates the power of these approaches. Finally, we will have to come back to classic biochemical and/or proteomic approaches to precisely define the molecular mechanisms how a transcription factor complex such as APl influences the decision of a cell to divide, differentiate, or die.

\section{ACKNOWLEDGEMENTS}

The IMP is supported by Boehringer-Ingelheim and I thank PD for comments on the manuscript.

\section{Author's affiliations}

E F Wagner, Research Institute of Molecular Pathology (IMP), Dr Bohr-Gasse 7, A-1030 Vienna, Austria

Correspondence to: Dr E F Wagner; wagner@nt.imp.univie.ac.at

\section{REFERENCES}

1 Jochum W, Passegue E, Wagner EF. AP-1 in mouse development and tumorigenesis. Oncogene $2001 ; 20: 2401-12$.

2 Shaulian E, Karin M. AP-1 as a regulator of cell life and death. Nat Cell Biol 2002;4:p. E131-6.

3 Wagner EF, Karsenty G. Genetic control of skeletal development. Curr Opin Genet Dev 2001;1 1:527-32.

4 Karsenty G, Wagner EF. Reaching a genetic and molecular understanding of skeletal development. Dev Cell 2002:2:389-406.

5 McCabe LR, Banerjee C, Kundu R, Harrison RJ, Dobner PR, Stein L, ef al. Developmental expression and activities of specific fos and jun proteins are functionally related to osteoblast maturation: role of Fra- 2 and Jun D during differentiation. Endocrinology 1996;137:4398-408.

6 Sandberg M, Vuorio T, Hirvonen H, Alitalo K, Vuorio E. Enhanced expression of TGF-beta and c-fos mRNAs in the growth plates of developing human long bones. Development 1988;102:461-70.

7 Johnson RS, Spiegelman BM, Papaioannou V. Pleiotropic effects of a null mutation in the c-fos proto-oncogene. Cell 1992;71:577-86.

8 Wang ZQ, Ovitt C, Grigoriadis AE, Mohle-Steinlein U, Ruther U, Wagner EF. Bone and hematopoietic defects in mice lacking c-fos. Nature 1992;360:741-5

9 Wang ZQ, Grigoriadis AE, Mohle-Steinlein U, Wagner EF. A novel target cell for c-fos-induced oncogenesis: development of chondrogenic tumours in embryonic stem cell chimeras. EMBO J 1991;10:2437-50.

10 Thomas DP, Sunters A, Gentry A, Grigoriadis AE. Inhibition of chondrocyte differentiation in vitro by constitutive and inducible overexpression of the c-fos proto-oncogene. J Cell Sci 2000;1 13:43950.

11 Grigoriadis AE, Schellander K, Wang ZQ, Wagner EF. Osteoblasts are target cells for transformation in c-fos transgenic mice. J Cell Biol 1993; 122:685-701.

12 Jochum W, David JP, Elliott C, Wutz A, Plenk HJ, Matsuo K, et al. Increased bone formation and osteosclerosis in mice overexpressing the transcription factor Fra-1. Nat Med 2000;6:985-90.

13 Sabatakos G, Sims NA, Chen J, Aoki K, Kelz MB, Amling M, et al. Overexpression of delta FosB transcription factor(s) increases bone formation and inhibits adipogenesis. Nat Med 2000;6:985-90.

14 Schreiber M, Wang ZQ, Jochum W, Fetka I, Elliott C, Wagner EF. Placental vascularisation requires the AP-1 component fra 1. Development 2000; 127:4937-48

15 Wang ZQ, Kiefer F, Urbanek P, Wagner EF. Generation of completely embryonic stem cell-derived mutant mice using tetraploid blastocyst injection. Mech Dev 1997:62:137-45.

16 Okada S, Wang ZQ, Grigoriadis AE, Wagner EF, von Ruden T. Mice lacking c-fos have normal hematopoietic stem cells but exhibit altered B-cell differentiation due to an impaired bone marrow environment. Mol Cell Biol 1994; 14:382-90.

17 Jain J, Nalefski EA, McCaffrey PG, Johnson RS, Spiegelman BM, Papaioannou $\mathrm{V}$, et al. Normal peripheral T-cell function in c-Fos-deficient mice. Mol Cell Biol 1994; 14: 1566-74

18 Grigoriadis AE, Wang ZQ, Cecchini MG, Hofstetter W, Felix R, Fleisch $\mathrm{HA}$, et al. c-Fos: a key regulator of osteoclast-macrophage lineage determination and bone remodeling. Science 1994:266:443-8.

19 Matsuo K, Owens JM, Tonko M, Elliott C, Chambers TJ, Wagner EF. Fosl 1 is a transcriptional target of c-Fos during osteoclast differentiation. Nat Genet 2000;24:184-7

20 Fleischmann A, Hafezi F, Elliott C, Reme CE, Ruther U, Wagner EF. Fra-1 replaces c-Fos-dependent functions in mice. Genes Dev 2000; 14:2695-700.

21 Owens JM, Matsuo K, Nicholson GC, Wagner EF, Chambers TJ. Fra-1 potentiates osteoclastic differentiation in osteoclast-macrophage precursor cell lines. J Cell Physiol 1999; 179:170-8.

22 Takayanagi H, Kim S, Matsuo K, Suzuki H, Suzuki T, Sato K, et al. RANKL maintains bone homeostasis through c-Fos-dependent induction of interferon-beta. Nature 2002;416:744-9.

23 Behrens A, Sibilia M, David JP, Mohle-Steinlein U, Tronche F, Schutz G, et al. Impaired postnatal hepatocyte proliferation and liver regeneration in mice lacking c-jun in the liver. EMBO J 2002;21:1782-90. 